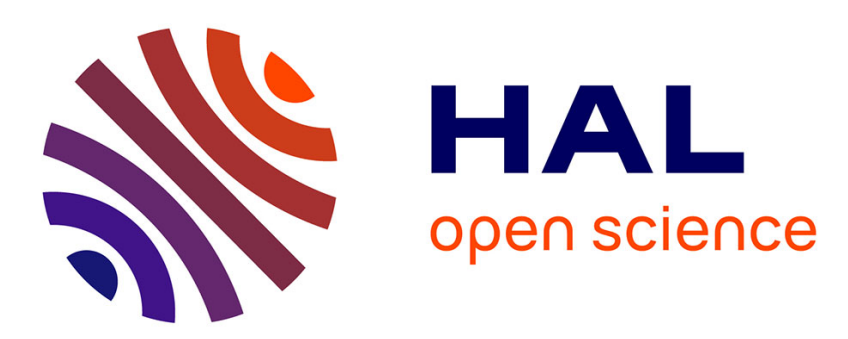

\title{
Boundary Control for Output Regulation in Scale-Free Positive Networks
}

Denis Nikitin, Carlos Canudas de Wit, Paolo Frasca

\section{To cite this version:}

Denis Nikitin, Carlos Canudas de Wit, Paolo Frasca. Boundary Control for Output Regulation in Scale-Free Positive Networks. CDC 2019 - 58th IEEE Conference on Decision and Control, Dec 2019,

Nice, France. pp.1-6. hal-02335142

\section{HAL Id: hal-02335142 \\ https://hal.science/hal-02335142}

Submitted on 28 Oct 2019

HAL is a multi-disciplinary open access archive for the deposit and dissemination of scientific research documents, whether they are published or not. The documents may come from teaching and research institutions in France or abroad, or from public or private research centers.
L'archive ouverte pluridisciplinaire HAL, est destinée au dépôt et à la diffusion de documents scientifiques de niveau recherche, publiés ou non, émanant des établissements d'enseignement et de recherche français ou étrangers, des laboratoires publics ou privés. 


\title{
Boundary Control for Output Regulation in Scale-Free Positive Networks
}

\author{
Denis Nikitin ${ }^{1}$, Carlos Canudas-de-Wit ${ }^{1}$ and Paolo Frasca ${ }^{1}$
}

\begin{abstract}
This paper deals with the problem of controlling the scalar output of a large-scale positive scale-free network to a constant reference value. We design an output-feedback controller such that no information about state vector or system matrices is needed. This controller can have arbitrary positive gains, and only one sufficient sign condition on system matrices should be satisfied. This controller can be used to regulate average state in a large-scale network with control applied to boundary nodes of the domain.
\end{abstract}

\section{INTRODUCTION}

Control of large-scale network systems is a very challenging but important task in a modern control theory due to its applications in dealing with many systems of interest such as urban traffic networks, social interactions, robot swarms or power networks.

This task is very difficult in the first place because of its large dimensionality, since in real-world applications the number of states can reach millions. The calculation of traditional control algorithms in this case is too expensive. In systems of such dimensions, the number of sensors is often much less than the number of states, as a result of which state feedback is not possible. Moreover, it is often not possible to identify the mathematical model of the system. Another difficulty is the amount of energy needed to control all nodes of the network, since as the number of elements increases, the energy needed to control them all grows exponentially for some network structures [6], [13]. In assessing energy efficient control problem it has been shown that several aspects such as network centrality [14] and number of control nodes [15] plays a fundamental role, but still energy analysis of large-scale network controllability is a very hard task to perform [16].

Therefore, in some cases, it can be preferrable to control some aggregated characteristics of the entire network rather than all individual states. For example, the control of the output of large-scale networks was studied in [7], [10]. The energy required to control aggregated characteristics instead of all network states is much less. A particular case of controlling the average state of large-scale networks in optimal way is studied in [8].

In this paper, having in mind the average control problem, we present the output regulation problem of a large linear network system under the assumption that it is stable and positive (that is, the system matrix has positive elements

*This work was supported by the funding from the European Research Council (ERC) under the European Union's Horizon 2020 research and innovation programme (grant agreement 694209)

${ }^{1}$ Univ. Grenoble Alpes, CNRS, Inria, Grenoble INP, GIPSA-Lab, Grenoble, France outside the main diagonal). Most network systems with stable dynamics and positive edge weights belong to this class. Positive systems are an important class of systems for which the synthesis of large-scale control algorithms can be greatly simplified. Their impulse response is bounded by their static gain [3], optimal and robust feedback control laws can be easily designed using linear programming ([4] and [5] respectively), and state feedback output regulation problem can be explicitly solved [2].

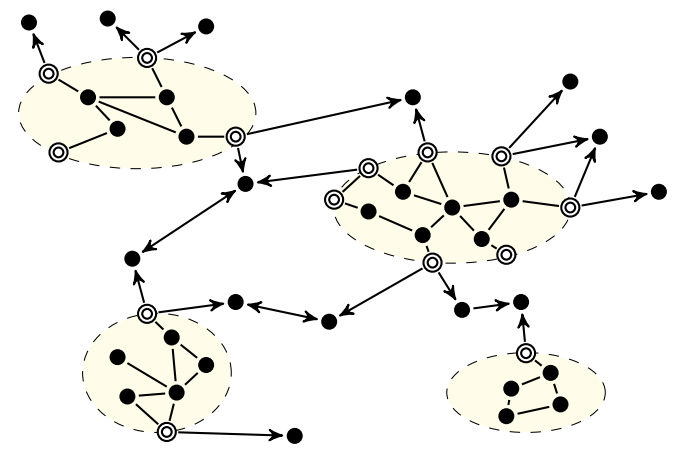

Fig. 1. Scale-Free network with hubs (shaded in yellow) controlled from the boundary nodes (double circles)

In opposition to the cited works we suppose that neither the measurement of all states, nor the control of all states is a necessary assumption of our network system. It is assumed that the only value that is measured and regulated is the value of the system output itself. Moreover, the system model is not used in the controller. Thus the equilibrium of internal states is never computed explicitly, and the controller directly utilizes only system output and reference point. Furthermore, the control can be applied to many network elements.

A particular example of such setup is a scale-free network, where the goal is to control the averaged state of the hubs and the control is applied to the boundaries of the hubs (see Fig. 1). Scale-free control of large-scale networks lies in the direction of the research project ERC Scale-FreeBack [9]. Output controllability of a scale-free network is studied in [10], the dual problem of reconstruction of an averaged state is solved in [11], and the reduction of a network to a scalefree is presented in [12].

The main contribution of our work is a simple sufficient sign condition on the system matrices that guarantees stability of any positive integral controller for controlling the system output to a constant reference point without knowledge of the system matrices. 
From the passivity analysis in the classical control theory it is known that the feedback interconnection between a linear operator with an integral controller is stable irrespectively of gain (has an infinite gain margin) if the linear operator is strictly positive real (SPR) [17]. From this point of view our work provides a new simple sufficient condition for the positive system to be SPR.

Notation: In our work several types of vector inequalities are used. For $x \in \mathbb{R}^{n}$ :

$$
\begin{aligned}
& x \geqslant 0 \Rightarrow \forall i \in\{1 . . n\}: x_{i} \geqslant 0, \\
& x>0 \Rightarrow \forall i \in\{1 . . n\}: x_{i} \geqslant 0 \text { and } \exists j \in\{1 . . n\}: x_{j}>0, \\
& x \gg 0 \Rightarrow \forall i \in\{1 . . n\}: x_{i}>0 .
\end{aligned}
$$

\section{Problem Formulation}

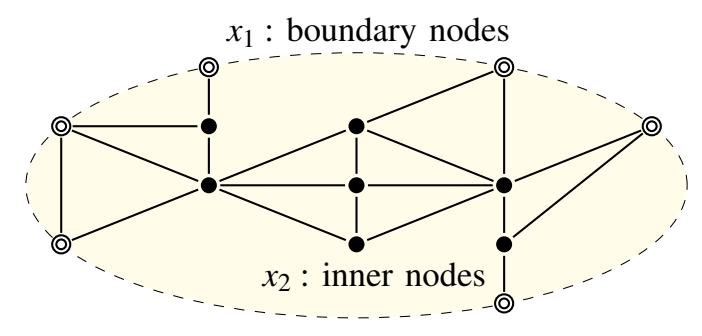

Fig. 2. Network with boundary and inner nodes separation

We start the problem formulation with an example: assume the system we need to control is the network given by the graph $\mathscr{G}=(\mathscr{V}, \mathscr{E})$, where $\mathscr{V}$ is the set of vertices and $\mathscr{E}$ is the set of edges. The number of vertices $|\mathscr{V}|$ is denoted by $n$.

On each node $v_{i} \in \mathscr{V}$ the state $x_{i}$ is defined. Each edge $e \in \mathscr{E}$, where $e=\left\{v_{i}, v_{j}\right\}$, corresponds to the flow between nodes $v_{i}$ and $v_{j}$. Matrix $A \in \mathbb{R}^{n \times n}$ represents exchange ratio. The set of nodes is split into two parts, boundary nodes $\mathscr{V}_{1}$ and inner nodes $\mathscr{V} / 2$ with state vectors $x_{1}$ and $x_{2}$ respectively (see Fig. 2). Assume that only flows to the boundary road sections can be controlled, thus control action $u$ is applied only to $x_{1}$ states. The mean state $y=\mathbf{1}^{T} x / n$ is measured. Thus the network depicted in Fig. 2 can be viewed as one particular hub from the scale-free network in Fig. 1.

The evolution of states $x$ and mean state $y$ is given by the following linear time-invariant system

$$
\left\{\begin{array}{l}
\dot{x}_{1}=A_{11} x_{1}+A_{12} x_{2}+u, \\
\dot{x}_{2}=A_{21} x_{1}+A_{22} x_{2}, \\
y=\frac{1}{n} \mathbf{1}^{T} x
\end{array}\right.
$$

The control goal is to stabilize mean state $y$ over the whole network to some desired constant state $y_{d}$ without the explicit knowledge of matrix A. It is assumed that the number of states is too large that it is impossible to use full-state feedback or to use matrix $A$ explicitly.

Most of real-world networks are internally stable, so we further assume $A$ being stable. Also we assume $A$ is a
Metzler matrix (which means its off-diagonal elements are non-negative), which means all edges have positive weights. Such choice of system matrix means that the system (1) belongs to the class of positive systems.

In the following more general case (2) is analysed, with general Metzler stable matrix $A \in \mathbb{R}^{n \times n}$, positive row-vector $C \in \mathbb{R}^{1 \times n}$ and general matrix $B \in \mathbb{R}^{n \times k}$, where the control goal is to stabilize system output $y$ to the desired constant value $y_{d}$ :

$$
\left\{\begin{array}{l}
\dot{x}=A x+B u \\
y=C x
\end{array}\right.
$$

\section{StRUCTURE OF MISO CONTROLlER}

Define transfer function of the system (2)

$$
W_{s}(s)=C(s I-A)^{-1} B,
$$

thus $y(s)=W_{s}(s) u(s)$. Denote error between desired output and system output: $e=y_{d}-y$. Then we can define controller transfer function $W_{c}(s)$ such that $u(s)=W_{c}(s) e(s)$. System control loop is depicted in Fig. 3.

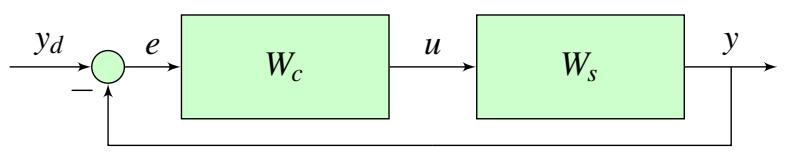

Fig. 3. Control loop

Thus input-output equation is

$$
y(s)=W_{s}(s) W_{c}(s) e(s),
$$

or, solving for $y$,

$$
y(s)=\frac{W_{s}(s) W_{c}(s)}{1+W_{s}(s) W_{c}(s)} y_{d} .
$$

Define closed-loop transfer function

$$
W(s)=\frac{W_{s}(s) W_{c}(s)}{1+W_{s}(s) W_{c}(s)} .
$$

In the following we investigate what properties do $W_{s}$ and $W_{c}$ have and what properties should $W$ have in order to be stable. Both $W_{s}$ and $W_{c}$ are vector-valued, because controller input $e$ and system output $y$ are scalars, while $u$ which is controller output and system input is a vector, $u \in \mathbb{R}^{k}$. Let us look at the $i$-th component of $W_{s}$ and $W_{c}$, where $i \in\{1 . . k\}$, and define polynomials $\alpha(s), \beta_{i}(s), \delta(s), \gamma_{i}(s)$ such that

$$
W_{s}(s)_{i}=\frac{\beta_{i}(s)}{\alpha(s)} \quad \text { and } \quad W_{c}(s)_{i}=\frac{\gamma_{i}(s)}{\delta(s)}
$$

It is obvious that $\alpha(s)$ is a polynomial of degree $n$. Moreover, the system is strictly stable and $y$ does not directly depend on $u$, thus

$$
\operatorname{deg} \beta_{i}(s)<\operatorname{deg} \alpha(s)=n \quad \text { and } \quad \alpha(s) \neq 0 \forall s: \operatorname{Re} s \geqslant 0 .
$$

We can choose $\alpha(s)$ and $\beta_{i}(s)$ such that $\alpha(s) \in \mathbb{R}$ for $s \in \mathbb{R}$ and $\alpha(s)>0$ for $s \geqslant 0$. 
Then, the controller $W_{c}$ should also be stable and casual, which means

$$
\operatorname{deg} \gamma_{i}(s) \leqslant \operatorname{deg} \delta(s) \quad \text { and } \quad \delta(s) \neq 0 \forall s: \operatorname{Re} s>0 .
$$

Again, it is possible to choose $\delta(s)$ and $\gamma_{i}(s)$ such that $\delta(s) \in$ $\mathbb{R}$ for $s \in \mathbb{R}$ and $\delta(s)>0$ for $s>0$.

Now we can rewrite $W(s)$ in terms of polynomials:

$$
W(s)=\frac{\sum_{i} \beta_{i}(s) \gamma_{i}(s)}{\alpha(s) \delta(s)+\sum_{i} \beta_{i}(s) \gamma_{i}(s)} .
$$

The closed-loop transfer function $W(s)$ should have the following property: for the constant input $y_{d}$ in should give the same output $y$, thus $W(0)=1$. This means that $\alpha(0) \delta(0)=0$, which is possible only if $\delta(0)=0$.

The simplest possible controller, satisfying this necessary condition, is the integral controller given by

$$
W_{c}(s)_{i}=\kappa \frac{\gamma_{i}}{s}
$$

where $\gamma \in \mathbb{R}^{k}$ is the vector of gains, defining relative control force applied to different actuated nodes, and $\kappa$ is the overall gain. The rest of the paper will be devoted to the integral controller and its properties.

\section{Stability of THE InTEGRAL CONTROLLER}

Assume we apply the integral controller (11) to system (2). The closed-loop system may be unstable, and in general in order to prevent this one needs to carefully choose controller gains in (11). It appears although that there exists simple criteria on the system matrices which says whether the closed-loop system will converge irrespectively of controller gain values, provided that they are positive. This result is the main contribution of this paper, and it is formulated as follows:

Theorem. The system (2) with applied integral controller (11) is asymptotically stable for any controller gains $\kappa>0$ and $\gamma$ if $C A^{2}>0$ and $C A^{2} B \gamma>0$.

Example (Damped consensus). Assume the system (2) is given by matrices $A=-L-\alpha I$, where $L$ is a Laplacian matrix of some network with $n$ nodes, $\alpha>0$ means additional damping to the system to preserve stability, and $C=\mathbf{1}^{T} / n$ represents average state of the network. Then $A$ is a Metzler stable matrix, and $C$ is the eigenvector of $A$ with corresponding eigenvalue $-\alpha$, thus $C A^{2}=\alpha^{2} C>0$. Then any controller with gains $\kappa>0$ and $\gamma$ will lead to the convergence, provided $B \gamma>0$.

One should notice that the condition $B \gamma>0$ on the control matrix $B$ is very non-restrictive, because by choosing appropriate vector gain $\gamma$ it is always possible to make $B \gamma>0$, and hence, provided $C A^{2}>0$ and $C A^{2} B \neq 0$, we will have $C A^{2} B \gamma>0$. The reason for this is the fact that the regulation variable is a single scalar output.

Corollary 1. It is possible to control the graph by any single boundary node as long as $C A^{2} \gg 0$.
Before proving the main theorem, we need to introduce the notion of M-matrix and state three technical lemmas.

Definition 1 ([1]). An $n \times n$ matrix $M$ that can be expressed in the form $M=\alpha I-L$, where $L=\left(l_{i j}\right)$ with $l_{i j} \geqslant 0,1 \leqslant$ $i, j \leqslant n$, and $\alpha \geqslant \lambda(L)$ where $\lambda(L)$ is the maximum of the moduli of the eigenvalues of $L$, is called an M-matrix.

From this definition it follows immediately that a negative of a Metzler stable matrix is an M-matrix. The main property of any M-matrix $M$ is that its inverse $M^{-1}$ is a positive matrix, thus $\left(M^{-1}\right)_{i j} \geqslant 0$ for all $i, j$.

Lemma 1. Suppose we have a matrix $\mathscr{M}=M+i b I$, which is a complex matrix with real part $M$ and imaginary part $b I$, with $b \in \mathbb{R}$ and $I$ an identity matrix. Assume $M$ being invertible and having no eigenvalues on the imaginary axis. Denote $\mathscr{L}=\mathscr{M}^{-1}=L+i \bar{L}$. Then the real part of $\mathscr{L}$ is given by

$$
\operatorname{Re} \mathscr{L}=L=\left(M+b^{2} M^{-1}\right)^{-1} .
$$

Lemma 2. Let $M$ be an M-matrix. Let $C$ be a row-vector such that $C M^{2}>0$. Then

$$
C\left(M+t M^{-1}\right)^{-1}>0
$$

for any $t \geqslant 0$.

Lemma 3. Let $M$ be an M-matrix. Let $C$ be a row-vector such that $C M^{2}>0$ and $C M^{2} B \gamma>0$. Then

$$
C\left(M+t M^{-1}\right)^{-1} B \gamma>0
$$

for any $t \geqslant 0$.

Proof. See proofs for the lemmas in the extended version of the paper in [18].

Proof of the theorem. Applying the integral controller and multiplying numerator and denominator by $s$, the transfer function of the closed-loop system is given by

$$
W(s)=\frac{\kappa C(s I-A)^{-1} B \gamma}{s+\kappa C(s I-A)^{-1} B \gamma} .
$$

For the stability of the closed-loop system $W(s)$ should have no poles on the right-hand side of the complex plane $\operatorname{Re} s>0$. Poles of $W(s)$ are given by roots of denominator. It is sufficient to show that the real part of denominator is strictly greater than zero in the right half-plane. Since $\operatorname{Re} s>0$ in the right half-plane, it is enough to show that $\operatorname{Re}\left\{\kappa C(s I-A)^{-1} B \gamma\right\}>0$.

Denote $\operatorname{Re} s=\alpha$ and $\operatorname{Im} s=\beta$, so matrix $(s I-A)^{-1}=$ $((\alpha I-A)+i \beta I)^{-1}$. Denote $M=\alpha I-A$. Matrix $A$ is a Metzler stable matrix, thus $(-A)$ is an M-matrix and matrix $M$ is an M-matrix too. Moreover, condition $C A^{2}>0$ implies $C M^{2}>0$ and $C A^{2} B \gamma>0$ implies $C M^{2} B \gamma>0$. Applying Lemma 1 we conclude that

$$
\operatorname{Re} \kappa C(M+i \beta I)^{-1} B \gamma=\kappa C\left(M+\beta^{2} M^{-1}\right)^{-1} B \gamma \text {. }
$$

By Lemma $3 C\left(M+t M^{-1}\right)^{-1} B \gamma>0$ for any $t \geqslant 0$, and assuming $\kappa>0$ we trivially obtain a sufficient condition on positivity of the real part of the denominator. 


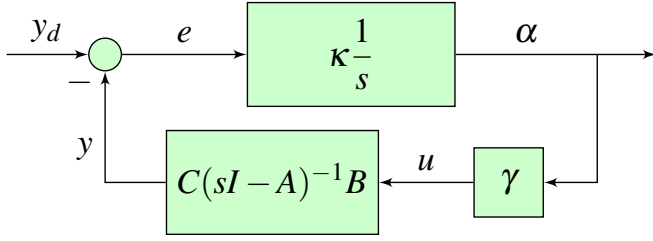

Fig. 4. Feedback interconnection of passive systems

Remark 1. An alternative proof of Theorem 1 can be also obtained via passivity arguments. Assume we fix an input gain vector $\gamma$ and define a new controller output $\alpha$ such that $u=\gamma \alpha$. Then the system (2) becomes SISO with respect to input variable $\alpha$. Define $H_{1}(s)=\kappa / s$ and $H_{2}(s)=C(s I-$ $A)^{-1} B \gamma$. It is possible to construct control loop with feedback interconnection as depicted in Fig. 4. The closed-loop system input is defined as $y_{d}$ and the system output is $\alpha$.

It is known that for $L_{2}$ stability of the system with feedback interconnection it is sufficient that the transfer function of one of the blocks is positive real ( $\mathrm{PR}$, which is equivalent to passivity) and another is strictly positive real (SPR) [17].

Passivity of an integral controller $H_{1}(s)$ is obvious, and Lemmas 1,2 and 3 are used to prove that $H_{2}(s)$ is SPR. Therefore the closed-loop system is $L_{2}$ stable.

Now it remains to prove that $y \rightarrow y_{d}$, which is obvious if one recalls that an output of a stable system with constant input converges to a constant value, thus for any constant $y_{d}$ there exists $\alpha^{*}$ such that $\alpha \rightarrow \alpha^{*}$. But convergence of an output of an integral controller means that its input converges to zero, which reads as $e \rightarrow 0$, which is exactly $y \rightarrow y_{d}$.

\section{NeCESSITY OF $C A^{2}>0$ CONDITION}

If $A$ is a Metzler stable matrix, all elements of $A^{-1}$ are nonpositive, thus condition $C A^{2}>0$ implies $C A<0$, which now implies $C>0$. The condition $C A^{2}>0$ is new and it is used in the Lemmas 2 and 3 (substituting $M=\alpha I-A$ as in the proof of the theorem). When one looks at the statement of the Lemma 2, one might think that it would be enough to require less restrictive condition $C A<0$ (which is obtained from the statement of the Lemma 2 by letting $t \rightarrow+\infty$ ). Moreover, the condition $C A<0$ has been proposed for the full state static feedback output control of positive systems by [2].

However, let us show that condition $C A^{2}>0$ is significant and $C A<0$ is not sufficient. An example of the positive system with $C A<0$ but $C A^{2} \ngtr 0$ would be

$$
A=\left(\begin{array}{ccc}
-1 & 0 & 0 \\
1 & -1 & 0 \\
0 & 1 & -1
\end{array}\right), \quad B=\left(\begin{array}{ll}
1 & 0 \\
0 & 1 \\
0 & 0
\end{array}\right), \quad C=\mathbf{1}^{T} .
$$

For this system $C A=(0,0,-1)$, but $C A^{2}=(0,-1,1)$. Now taking $s=0.01+2 i$ results in

$$
\operatorname{Re} C(s I-A)^{-1}=\left(\begin{array}{lll}
-0.0047 & 0.0829 & 0.2012
\end{array}\right) .
$$

First component of this vector is negative, thus result of theorem 1 is violated. Moreover, choosing control vector $\gamma=(1 ; 0)$ (and thus controlling only the first node) leads to the unstability of the closed-loop system for $\kappa=3$ (although with $\kappa=2$ the system is still stable). This confirms our understanding that the novel $C A^{2}>0$ condition is meant to ensure stability using any arbitrary boundary node and arbitrary positive gain $\kappa$.

\section{TOPOLOGICAL INTERPRETATION OF $C A^{2}>0$}

To understand topological properties of the condition $C A^{2}>0$ we first start with more intuitive one, $C A<0$, which is implied by $C A^{2}>0$.

Define matrices $D$ and $E$ such that $A=E-D$, with $D$ being diagonal and $E$ having all diagonal elements zero. Thus both $D$ and $E$ have all their entries positive. Matrix $E$ can be viewed as adjacency matrix of the network, with element $E_{i j}$ meaning influence of node $v_{j}$ on node $v_{i}$. Matrix $D$ consists of the self-damping powers on the diagonal. Therefore condition $C A<0$ reads as $C D>C E$. This condition states some kind of diagonal dominance in the network.

Assume some $C_{i}=0$. Then $(C D)_{i}=0$ because $D$ is diagonal. Thus $(C E)_{i}$ should be also zero, which means that for every index $j$ either $C_{j}=0$ or $E_{j i}=0$.

Corollary 2. If node $v_{i}$ is not included in the aggregated output $\left(C_{i}=0\right)$, then its reachable set should not be included either.

- For a strongly connected graph this means that all nodes should be included in the aggregated output.

- If the network is divided into "boundary" nodes and "inner" nodes, and the goal is to control the average of the inner nodes, then at least one of the boundary nodes should also be included into the average.

In the same manner it is possible to see this condition as a lower bound on the damping of each node: $D_{i i} \geqslant \sum_{j} C_{j} E_{j i} / C_{i}$. Thus the bigger is the influence of the node's neighbours in the output, the bigger should be the node's damping.

We can use the same decomposition $A=E-D$ in order to understand the condition $C A^{2}>0$ and conclude that

$$
C E^{2}+C D^{2}>C(E D+D E) .
$$

Being quadratic inequality, this condition bounds damping of each node from above and below with respect to dampings of other nodes. We will see examples in the following section.

\section{EXAMPLES}

\section{A. Network with star topology}

In this section we present several examples of networks and analyse the condition $C A^{2}>0$ for them. To begin with we choose network with star topology with one central node and $n$ leafs, average state of which we want to control. Let nodes $1 \ldots n$ be the leafs and node $n+1$ be the hub. Assume central hub and the first leaf belong to the boundary node set and thus can be controlled (see Fig. 5). 


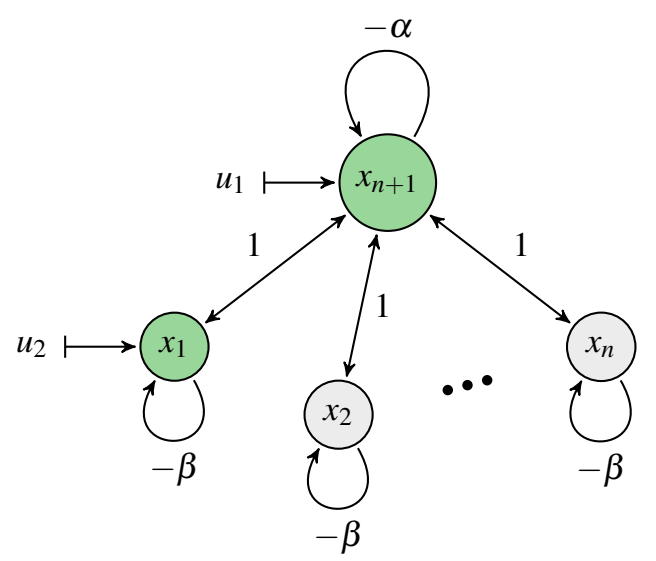

Fig. 5. Network with star topology with $n$ leafs. Boundary nodes in green.

Dynamics of this network can be written as system (2) with matrices

$$
\begin{gathered}
A=\left(\begin{array}{cccc}
-1-\beta & 0 & \cdots & 1 \\
0 & -1-\beta & \cdots & 1 \\
\vdots & \vdots & \ddots & \vdots \\
1 & 1 & \cdots & -n-\alpha
\end{array}\right), \quad B=\left(\begin{array}{cc}
0 & 1 \\
0 & 0 \\
\vdots & \vdots \\
1 & 0
\end{array}\right), \\
C=\mathbf{1}^{T} /(n+1) .
\end{gathered}
$$

Such choice of system matrices corresponds to the undirected network with star topology and damping $\alpha>0$ for central node and $\beta>0$ for all other nodes. Integral controller (11) with $\gamma=(1,0)^{T}$ would correspond to the control applied only to the hub, and controller with $\gamma=(0,1)^{T}$ would correspond to the control of the first leaf.

Calculating $C A$ and $C A^{2}$ gives

$$
\begin{aligned}
& C A=\left(\begin{array}{llll}
-\beta & -\beta & \cdots & -\alpha
\end{array}\right) /(n+1)<0, \\
& \left(C A^{2}\right)_{1 \ldots n}=\left(\beta^{2}+(\beta-\alpha)\right) /(n+1), \\
& \left(C A^{2}\right)_{n+1}=\left(\alpha^{2}+n(\alpha-\beta)\right) /(n+1) .
\end{aligned}
$$

$C A^{2}>0$ means then $\alpha^{2}+n(\alpha-\beta) \geqslant 0$ and $\beta^{2}+(\beta-\alpha) \geqslant$ 0 with at least one of these inequalities being strict. Solving this for damping of leaf nodes we obtain

$$
\sqrt{\alpha+\frac{1}{4}}-\frac{1}{2} \leqslant \beta \leqslant \alpha+\frac{\alpha^{2}}{n}
$$

thus $\beta$ is bounded from both sides with respect to $\alpha$. Moreover, as $n \rightarrow \infty$, we obtain limit inequality $\beta \leqslant \alpha$, which means that damping for leafs should be lower than for hubs.

Simulation results for both cases, $\gamma=(1,0)^{T}$ and $(0,1)^{T}$ and for $n=20$ leafs are given in Fig. 6, with dampings $\alpha=2$, $\beta=1.1$, desired output $y_{d}=5$ and controller gain $\kappa=12$. In Fig. $6 \mathrm{a}$ it is clearly seen that controlling either the root or the leaf has almost the same effect on the output $y$.

\section{B. Line network}

Now we explore an example of a directed line network with $n$ nodes. This network is depicted in Fig. 7. As usual, we are interested in controlling average state of the network, and it is assumed that we can control only the input node $x_{1}$ of the system.

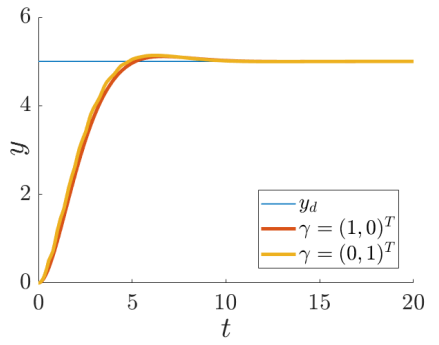

(a)

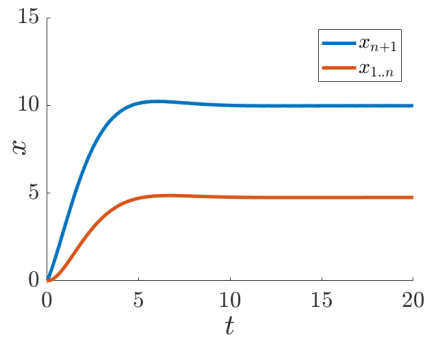

(b)
Fig. 6. Output control of the star network with $n=20$ leafs. $\alpha=2, \beta=1.1$, $\kappa=12, y_{d}=5$. (a). Output $y$ for different $\gamma$ vectors. (b). Spread of states $x$ for $\gamma=(1,0)^{T}$ corresponding to the control of the root. All the leaf states $x_{1} \ldots x_{20}$ have the same asymptotic value 4.751 (which is obvious from the symmetry), while the root state converges to 9.978 .

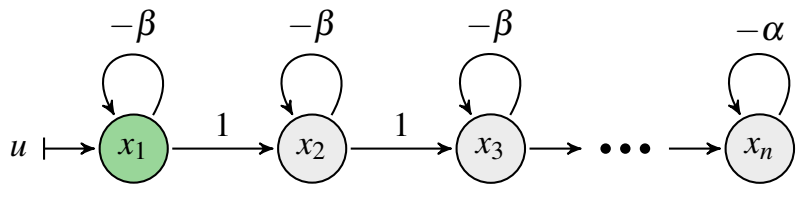

Fig. 7. Directed line network with $n$ nodes. Boundary node in green

System matrices for $n$ nodes are given as follows:

$$
\begin{gathered}
A=\left(\begin{array}{cccc}
-1-\beta & 0 & \cdots & 0 \\
1 & -1-\beta & \cdots & 0 \\
\vdots & \vdots & \ddots & \vdots \\
0 & 0 & \cdots & -\alpha
\end{array}\right), \quad B=\left(\begin{array}{c}
1 \\
0 \\
\vdots \\
0
\end{array}\right), \\
C=\mathbf{1}^{T} / n, \quad \gamma=1 .
\end{gathered}
$$

This choice of system matrices corresponds to the directed line network with damping $\alpha>0$ for the last node and $\beta>0$ for all other nodes.

Calculating $C A$ and $C A^{2}$ gives

$$
\begin{aligned}
& C A=\left(\begin{array}{llll}
-\beta & -\beta & \cdots & -\alpha
\end{array}\right) / n<0, \\
& \left(C A^{2}\right)_{1 . . n-2}=\beta^{2} / n>0, \\
& \left(C A^{2}\right)_{n-1}=\left(\beta^{2}+\beta-\alpha\right) / n, \\
& \left(C A^{2}\right)_{n}=\left(\alpha^{2}+\alpha-\beta\right) / n,
\end{aligned}
$$

$C A^{2}>0$ means then $\alpha^{2}+(\alpha-\beta) \geqslant 0$ and $\beta^{2}+(\beta-\alpha) \geqslant$ 0 . Solving this for damping of leaf nodes we obtain

$$
\sqrt{\alpha+\frac{1}{4}}-\frac{1}{2} \leqslant \beta \leqslant \alpha+\alpha^{2}
$$

thus $\beta$ is bounded from both sides with respect to $\alpha$.

In order to validate the conclusions about this example, we take directed line networks with 4 and 100 nodes and check whether they are stable or unstable for different $\kappa$.

Fix $\alpha=0.2$, therefore for condition $C A^{2}>0$ to hold one needs $\sqrt{0.45}-0.5 \leqslant \beta \leqslant 0.24$. In Fig. 8 simulation results are shown for $\kappa=12, y_{d}=5$ and for two values of $\beta$, the first, $\beta=0.2$, satisfies the condition, and the second $\beta=$ 0.002 does not. In the case $\beta=0.2$ and $n=100$ it is very interesting to see what are limit values of the state variables $x$. It appears that they decrease exponentially (due to the relation $\left.x_{i-1}-(1+\beta) x_{i}=0\right)$ starting from the controlled node $x_{1}$, while preserving their average equal to $y_{d}$. 


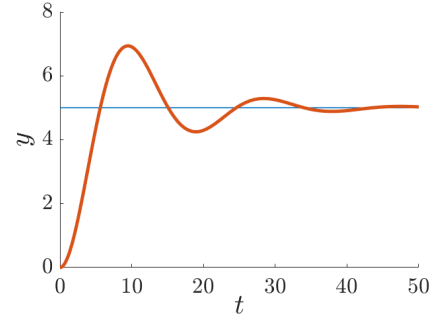

(a)

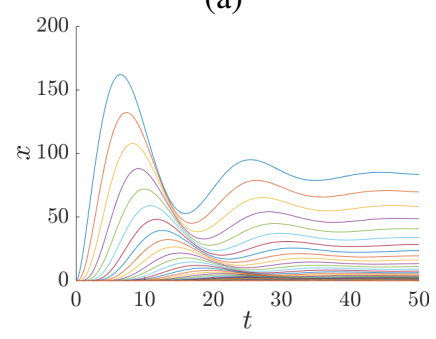

(c)

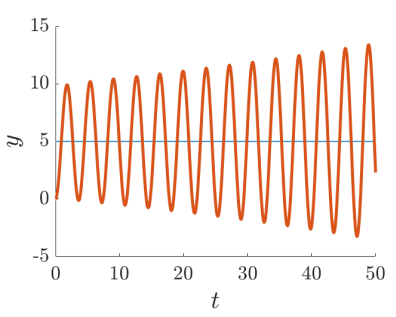

(b)

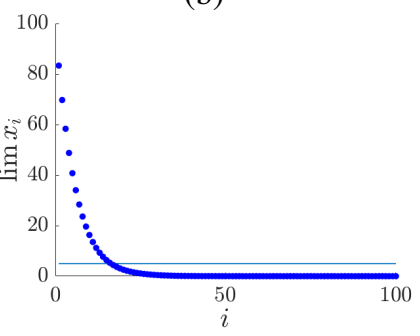

(d)
Fig. 8. Output control of the directed line network, $\alpha=0.2, \kappa=12, y_{d}=5$. Inset (b). $n=4, \beta=0.002$. Output $y$ of the network is unstable, $C A^{2} \ngtr 0$. Insets (a), (c), (d). $n=100, \beta=0.2$. Network is stable. (a). Output $y$. (c). Spread of states $x$. (d). Values of $\lim _{t \rightarrow \infty} x_{i}$ depending on the number $i \in$ $\{1 . .100\}$, which is the distance from the controlled node.

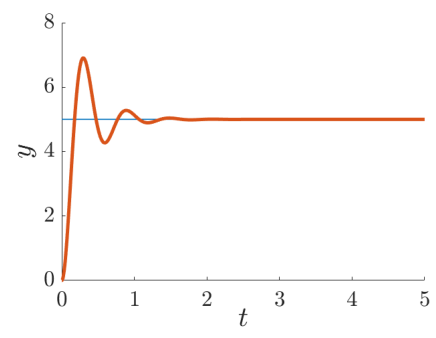

(a)

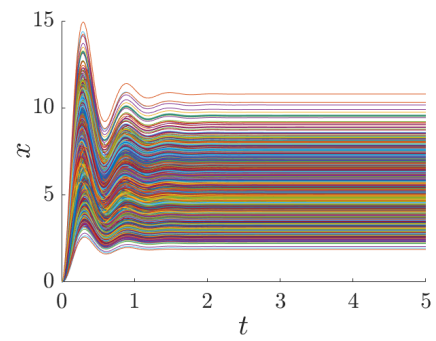

(b)
Fig. 9. Output control of Erdős-Rényi graph for $N=4000$ nodes, $\kappa=250$. (a). Dynamics of output $y$. (b). Dynamics of states $x$.

\section{Random Erdös-Rényi graph}

Here we present a simulation results for an integral controller for random Erdős-Rényi graph with $N=4000$ nodes and probability of creating an edge $p=0.01$. Vector $C=\mathbf{1}^{T} / N$ represents the average, and the system matrix $A$ is the negative of a Laplacian of this ER graph with an additional random damping on every node taken from the uniform distribution $U(6,7)$ such that $C A^{2}>0$. Matrix $B$ is chosen to be a random vector of zeros and ones with equal probability. With such setup for any $\kappa>0$ the system converged to the desired output reference $y_{d}=5$, see Fig. 9 .

\section{CONCLUSION}

In this paper we considered problem of output control of a large scale positive network system. We examined the general properties of the transfer functions of the system and the controller and determined the general form that the controller must satisfy in order to solve the problem of controlling the output to a given constant reference point. The integral controller was chosen as the simplest one satisfying the general form.
We formulated sufficient condition $C A^{2}>0$ for the convergence of any positive integral controller and gave an example showing the conservatism of this condition. If the system satisfies this condition, there is no need to adjust the parameters of the controller, to have knowledge of the state vector or the values of the elements of the $A$ matrix.

We showed that satisfaction of this condition for a positive system means that all network node states should be included in the aggregated output, and moreover, this condition imposes restrictions on the relative values of damping between neighbouring nodes, which is especially important in the case of different degrees of these nodes, for example as in scalefree networks.

\section{ACKNOWLEDGement}

The Scale-FreeBack project has received funding from the European Research Council (ERC) under the European Union's Horizon 2020 research and innovation programme (grant agreement N 694209).

\section{REFERENCES}

[1] R. J. Plemmons. M-Matrix Characterizations. I - Nonsingular MMatrices. 1977.

[2] F. N. Nogueira. Contributions to positive systems control: mathematical methods and applications. PhD. Thesis. 2013.

[3] A. Rantzer. Distributed control of positive systems. arXiv:1203.0047. 2014.

[4] A. Rantzer. Scalable control of positive systems. European Journal of Control 24, 72-80. 2015.

[5] C. Briat. Robust stability and stabilization of uncertain linear positive systems via integral linear constraints: $L_{1}$-gain and $L_{\infty}$-gain characterization. Int. J. Robust Nonlinear Control 23, 1932-1954. 2013.

[6] G. Yan, J. Ren, Y. C. Lai, C. H. Lai, B. Li. Controlling complex networks: How much energy is needed. Physical review letters, 108(21), 218703. 2012.

[7] I. Klickstein, A. Shirin and F. Sorrentino. Locally Optimal Control of Complex Networks. Physical review letters, 119(26), 268301. 2017.

[8] L. Vassio, F. Fagnani, P. Frasca and A. Ozdaglar. Message Passing Optimization of Harmonic Influence Centrality. IEEE Transactions on Control of Network Systems, vol. 1, no. 1, pp. 109-120, March 2014.

[9] C. Canudas-de-Wit, ERC: Scale-Free Control for Complex Physical Network Systems, http://scale-freeback.eu.

[10] G. Casadei, C. Canudas-de-Wit, S. Zampieri. Scale Free Controllability of Large-Scale Networks: an Output Controllability Approach. CDC 2018 - 57th IEEE Conference on Decision and Control, Dec 2018, Miami, FL, United States. pp.1-8.

[11] M. U. B. Niazi, C. Canudas-de-Wit, A. Y. Kibangou. Average observability of large-scale network systems. European Control Conference (ECC), 2019, to appear.

[12] N. Martin, P. Frasca, C. Canudas-de-Wit. Large-scale network reduction towards scale-free structure. IEEE Transactions on Network Science and Engineering, IEEE, 2018, pp.1-12.

[13] Y. Y. Liu, J. J. Slotine, A. L. Barabasi. Controllability of complex networks. Nature 473 (7346), pp. 167-173, 2011.

[14] G. Lindmark, C. Altafini. A driver node selection strategy for minimizing the control energy in complex networks. IFAC-PapersOnLine, 50 (1), pp. 8309-8314, 2017.

[15] G. Yan, G. Tsekenis, B. Barzel, J. J. Slotine, Y. Y. Liu, and A. L. Barabasi. Spectrum of Controlling and Observing Complex Networks. Nature Physics, 2015.

[16] F. Pasqualetti, S. Zampieri, F. Bullo. Controllability Metrics, Limitations and Algorithms for Complex Networks. IEEE Trans. on Control of Net. Systems, 1(1), pp. 40-52, 2014.

[17] R. Sepulchre, M. Jankovic, P. V. Kokotovic. Constructive nonlinear control. Springer Science and Business Media. 2012.

[18] D. Nikitin, C. Canudas-de-Wit, P. Frasca. Control of Average and Variance in Large-Scale Linear Networks. In preparation. 